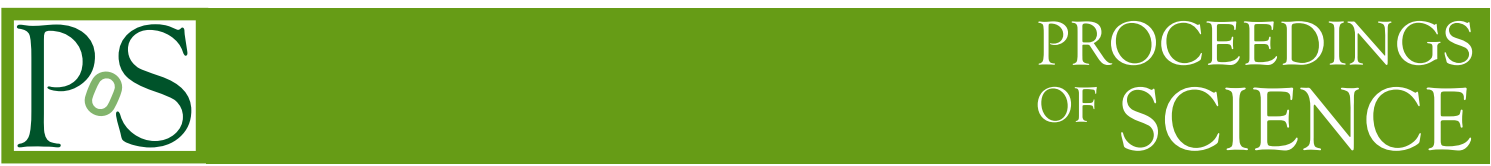

\title{
AKARI Contribution to the Dust Studies
}

\author{
Issei Yamamura* \\ Institute of Space and Astronautical Science(ISAS), JAXA, Japan \\ E-mail: yamamura@ir.isas.jaxa.jp
}

\section{and $A K A R I$ Team}

\begin{abstract}
$A K A R I$ is the first Japanese satellite mission dedicated for the infrared (IR) astronomy. In operation from 2006 to 2011, AKARI has carried out an all-sky survey as well as twenty thousand pointed observations from near- to far-IR wavelengths over four years of science operation. The All-Sky far- and mid-IR Point Source Catalogues, Asteroid catalogue, as well as point source catalogues from the Large Magellanic Cloud and the North Ecliptic Pole region surveys have been published. The AKARI team is still working on data processing and archiving. The far-IR All-Sky Survey Point Source Catalogue, mid- and far-IR Faint Source Catalogues, all-sky image maps are currently being revised. Imaging and spectroscopic data taken in the pointed observation mode will also be archived as "science ready" data products. The broad wavelength coverage and sky coverage of the AKARI data shall contribute to research in cosmic dust.
\end{abstract}

The Life Cycle of Dust in the Universe: Observations, Theory, and Laboratory Experiments - LCDU 2013, 18-22 November 2013

Taipei, Taiwan

\footnotetext{
* Speaker.
} 


\section{The AKARI Mission}

The first Japanese infrared astronomical satellite AKARI [6] was launched on February 22, 2006 (JST). AKARI was equipped with a $68.5 \mathrm{~cm}$ telescope cooled down to below $7 \mathrm{~K}$ and two scientific instruments, the Far-infrared Surveyor (FIS; [3]) and the Infrared Camera (IRC; [8]). The FIS covered the wavelength range between $50-180 \mu \mathrm{m}$ by two broad and two narrow bands. A Fourier Transform Spectrometer (FTS) enabled us to carry out imaging-spectroscopic observations in the same wavelength range. The IRC observed with three cameras, namely the NIR $(1.8-5.3 \mu \mathrm{m})$, the MIR-S $(5.4-13.1 \mu \mathrm{m})$ and the MIR-L $(12.4-26.5 \mu \mathrm{m})$. Each camera had three filter bands and grism/prism for low-resolution spectroscopy.

AKARI had two observing modes; the survey mode and the pointing mode. The former was used for the All-Sky Survey. The FIS and IRC MIR-S\&L were operated and scanned the sky with a speed of $\sim 3.6 \mathrm{arcmin} / \mathrm{sec}$. The pointing mode was used for deep imaging and spectroscopic observations of particular targets. A pointing enabled about 12 minutes exposure with a cost of total 30 minutes including maneuver etc.

The scientific operation started on May 2006. The first half year was mainly dedicated to the All-Sky Survey. Only a small number of pointed observations were carried out in the highvisibility (i.e., high ecliptic latitude) regions. In the following period until the liquid helium boil-off in August, 2007, supplemental All-Sky Survey and thousands of pointed observations were carried out. The warm mission started in June 2008 with only the NIR channel of the IRC, cooled with the cryocoolers. Thirteen thousand imaging and spectroscopic observations were carried out until February 2010, when the degradation of the cryocoolers prevent to take sufficient quality data.

\section{AKARI Data Products}

A huge amount of data taken by AKARI are legacy to future astronomical research and must be archived so that the world-wide astronomical community can easily access the information.

The first products from the AKARI All-Sky Survey were the point source catalogues. The IRC Point Source Catalogue Ver.1 [1] and the FIS Bright Source Catalogue Ver.1 [12] were released in March 2010. The IRC catalogue contains 870,973 sources, and the FIS catalogue presents 427,071 objects. Interestingly, we find that only 20,000-30,000 sources (depending on search radius) matches between the two catalogues within positional errors. This emphasizes the difference in the populations contained in the two catalogues.

A catalogue of 5120 asteroids from the mid-IR All-Sky Survey data was constructed [11]. The catalogue lists the size and albedo of the objects calculated from the AKARI fluxes using the Standard Thermal Model of the asteroids. It is the most complete public catalogue at this moment, and enables us to make more precise statistical studies of asteroids.

The North Ecliptic Pole region survey resulted in the point source catalogues [10, 4, 7]. The catalogues are extensively used for the extragalactic science, for instance studies of the galaxy evolution. Thanks to the continuous wavelength coverage of the AKARI/IRC filters, precise SED analysis becomes possible.

Two products have been published from the Large Magellanic Cloud (LMC) survey. The AKARI-LMC point source catalogue [2] contains 660 thousand of point sources detected in the 
near- and mid-IR. This is a complimentary survey to Spitzer-SAGE survey [5]. The near-IR spectra of 1757 sources observed in the LMC survey were extracted [9]. These catalogues shall be useful for classification of the objects as well as studies of the gas and solid state features of young and evolved stars.

The AKARI data products so far released to public are available at the AKARI observer web

Table 1: Planned AKARI Processed Data Products

\begin{tabular}{|c|c|c|c|}
\hline Product name & Description & Priority & Public Release \\
\hline $\begin{array}{l}\text { FIS Bright Source Cat- } \\
\text { alogue Ver.2 }\end{array}$ & $\begin{array}{l}\text { Revision of the FIS BSC. Accuracy } \\
\text { and reliability will be improved. } \\
\text { Single-scan photometric database } \\
\text { and scan density data will also be } \\
\text { available. }\end{array}$ & 1 & Mar. 2015 \\
\hline $\begin{array}{l}\text { FIS Faint Source Cata- } \\
\text { logue Ver.1 }\end{array}$ & $\begin{array}{l}\text { The catalogue improves detection } \\
\text { limit in the high-visibility regions. }\end{array}$ & 1 & June 2015 \\
\hline $\begin{array}{l}\text { FIS All-Sky image } \\
\text { maps }\end{array}$ & $\begin{array}{l}\text { All-Sky image maps in the four FIS } \\
\text { bands }(65,90,140,160 \mu \mathrm{m})\end{array}$ & 2 & Mar. 2016 \\
\hline $\begin{array}{l}\text { IRC Faint Source Cat- } \\
\text { alogue }\end{array}$ & $\begin{array}{l}\text { The catalogue improves detection } \\
\text { limit in the high-visibility regions. }\end{array}$ & 1 & Mar. 2016 \\
\hline $\begin{array}{l}\text { IRC All-Sky Image } \\
\text { Maps }\end{array}$ & $\begin{array}{l}\text { All-Sky image maps in the two IRC } \\
\text { bands }(9 \& 18 \mu \mathrm{m})\end{array}$ & 2 & Mar. 2016 \\
\hline $\begin{array}{l}\text { Asteroid Catalogue } \\
\text { Ver.2 }\end{array}$ & $\begin{array}{l}\text { Additional } 1000 \text { asteroids supple- } \\
\text { mental to the Ver. } 1 \text { are expected. }\end{array}$ & 5 & Mar. 2016 \\
\hline FIS Slow-scan Atlas & $\begin{array}{l}\text { Processed image data of individ- } \\
\text { ual FIS Slow-scan mapping obser- } \\
\text { vations. }\end{array}$ & 4 & Mar. 2016 \\
\hline FIS FTS data & $\begin{array}{l}\text { Processed imaging-spectroscopic } \\
\text { data-set observed with the Fourier } \\
\text { Transform Spectrometer (FTS) of } \\
\text { the FIS. }\end{array}$ & 5 & Sep. 2015 \\
\hline $\begin{array}{l}\text { IRC Pointed Observa- } \\
\text { tion Images }\end{array}$ & $\begin{array}{l}\text { Processed imaging (photometric) } \\
\text { observation data of the IRC. Indi- } \\
\text { vidual observation data will be pro- } \\
\text { cessed separately. No mosaicing } \\
\text { will be applied. }\end{array}$ & 4 & Mar. 2016 \\
\hline FIS Slow-scan Atlas & $\begin{array}{l}\text { Processed image data of individ- } \\
\text { ual FIS Slow-scan mapping obser- } \\
\text { vations. }\end{array}$ & 4 & Sep. 2015 \\
\hline $\begin{array}{l}\text { IRC Slit spectroscopy } \\
\text { Data }\end{array}$ & $\begin{array}{l}\text { Spectra taken with the IRC spec- } \\
\text { troscopic mode with slit (and point } \\
\text { source aperture mask). }\end{array}$ & 4 & Mar. 2016 \\
\hline $\begin{array}{l}\text { IRC Slitless spec- } \\
\text { troscopy Data }\end{array}$ & $\begin{array}{l}\text { Spectra taken with the IRC spectro- } \\
\text { scopic mode on the imaging field } \\
\text { with the objective grism/prism. }\end{array}$ & 5 & Mar. 2016 \\
\hline
\end{tabular}


page $^{1}$ and ISAS's data archive DARTS ${ }^{2}$. More than 1000 downloads of the All-Sky Catalogues have been made since the release. The catalogues are also available from other archives in the world, such as those at IPAC or CDS. On-line data search services are also provided at ISAS and other systems. As of January 2014, about 700 refereed science papers using AKARI data have been published, in which about 240 are based on the catalogue data.

Following the termination of the satellite operation, the main mission of the AKARI project team has shifted to the data processing and archiving. The activity continues until 2017. The goal of the project is to construct and archive "science ready" datasets for as many AKARI observations as possible. Table 1 describes the products we plan to provide. The data processing activities are prioritized by their potential impact on research and technical difficulties.

\section{Summary}

AKARI has completed the 2nd generation All-Sky Survey in the mid- and far-IR regime and produced the All-Sky Point Source Catalogues containing in total 1.3 million sources. Most of the sources in the catalogue have never been investigated in detail, and can possibly be critical objects that open a new door to astronomical research. AKARI's unique wavelengths and broad area surveys shall be useful for the dust studies. Readers of this article are welcome to visit the AKARI data archive.

\section{Acknowledgments}

$A K A R I$ is a JAXA project with the participation of ESA.

\section{References}

[1] Ishihara, D., Onaka, T., Kataza, H., et al. 2010, $A \& A$, 514, A1

[2] Kato, D., Ita, Y., Onaka, T., et al. 2012, AJ, 179, 179

[3] Kawada, F., Baba, H., Barthel, P. D., et al. 2007, PASJ, 59, S389

[4] Kim, S. J., Lee, H. M., Matsuhara, H., et al. 2013, A\&A, 548, A29

[5] Meixner, M., Gordon, K. D., Indebetouw, R., et al. 2006, AJ, 132, 2288

[6] Murakami, H., Baba, H., Barthel, P. D., et al. 2007, PASJ, 59, S369

[7] Murata, K., Matsuhara, H., Wada, T., et al. 2013, A\&A, 559, A132

[8] Onaka, T., Matsuhara, H., Wada, T., et al. 2007, PASJ, 59, S401

[9] Shimonishi, T., Onaka, T., Kato, D., et al. 2013, AJ, 145, 32

[10] Takagi, T., Matsuhara, H., Goto, T., et al. 2012, A\&A, 537, A24

[11] Usui, F., Kuroda, D., Müller, T. G., et al. 2011, PASJ, 63, 1117

[12] Yamamura, I., et al. 2010, AKARI/FIS All-Sky Survey Bright Source Catalogue Version 1.0 Release Note, http://www.ir.isas.jaxa.jp/AKARI/Observation/

\footnotetext{
${ }^{1}$ http://www.ir.isas.jaxa.jp/AKARI/Observation/

${ }^{2}$ http://darts.isas.jaxa.jp/ir/akari/
} 